\title{
Distribución del potencial electrostático en UNA PLACA CUADRAda UTILIZANDO EL MÉTOdO DE ELEMENTOS FINITOS
}

\author{
Jairo Madrigal Argáez ${ }^{1}$ \\ JaIMe Barbosa PÉREZ ${ }^{2}$ \\ Manuel Julio García ${ }^{3}$
}

\section{Resumen}

Este artículo expone la solución al problema de la distribución del potencial electrostático en una placa cuadrada mediante el Método de Elementos Finitos (Finite Elements Method, FEM). En este proceso se implementa el método pesaje residual en la formulación débil de la ecuación diferencial de Laplace con condiciones de frontera de Dirichlet para el potencial electrostático. La solución encontrada se basa en la selección de funciones lineales sobre un dominio discretizado en un número finito de elementos geométricos. La estrategia de solución se basa en la implementación del método de Galerkin en la escogencia de la función solución de la ecuación de Laplace llevada a su representación discreta.

\section{Palabras clave}

Flujo eléctrico, potencial electrostático, laplace, galerkin, elementos finitos.

1 Físico, Especialista en óptica técnica. Profesor de física de la facultad de Ciencias Básicas, ITM, Medellín, Colombia. jairomadrigal@itm.edu.co

2 Ingeniero Mecánico. Profesor del departamento de Ingeniería Mecánica, Universidad EAFIT, Medellín, Colombia, jbarbosa@eafit.edu.co.

3 Manuel Julio Garcia Ruiz. Director del grupo de mecánica computación Universidad EAFIT, Medellín, Colombia.mgarcia@eafit.edu.co 


\section{Abstract}

This paper presents the solution to the problem of the distribution of the electrostatic potential in a square plate by means of the Finite Elements Method (FEM). In this process the method is implemented minimo remainder in the weak formulation of the equation differential of Laplace with conditions of border of Dirichlet for the electrostatic potential. The found solution is based on the selection of linear functions on a dominion discreet in a finite number of geometric elements. The solution strategy is based on the implementation of the method of Galerkin in the choosing of the function solution of the equation of Laplace taken to its discreet representation.

\section{Key words}

Electric flow, electrostatic potential, Laplace solution, Galerkin solution, Finite Elements Method (FEM). 


\section{INTRODUCCIÓN}

El método de elementos finitos es una técnica de aproximación numérica que se viene empleando en la solución de problemas en una amplia gama de ambientes técnicos en ciencia e ingeniería donde soluciones numéricas son requeridas. En este documento se expone el Método de Elementos Finitos (FEM) de sus siglas en ingles, en la solución al problema de la distribución del potencial electrostático en una placa cuadrada con condiciones de frontera. El resultado de la aplicación de esta técnica es comparativamente aceptable con la solución analítica tradicionalmente expuesta en la literatura científica ${ }^{4}$.

El FEM se utiliza para desarrollar un procedimiento que permita ponderar una función solución de mínimo residuo o error, tal que se pueda determinar el comportamiento de una función en una región acotada. La elección de la función solución al problema se determina por el método de Galerkin; posteriormente, para efectos de la solución del problema propuesto, la ecuación del promedio residual es llevada a la forma discreta ${ }^{5}$ mediante la formulación débil y finalmente evaluada sobre un dominio discretizado en un número finito de elementos triangulares lineales. De esta manera se expone una solución al problema de la distribución del potencial electrostático en una placa cuadrada mediante la solución de la ecuación de Laplace con condiciones de frontera de Dirichlet. Los resultados obtenidos con esta técnica se comparan con la solución analítica y se calcula el error.

Este ejercicio se fundamenta en la formulación de la técnica de elementos finitos implementada en el libro Lecture Notes on Numerical Análisis por Manuel García [1] y en el libro The Finite Element Method Using Matlab por Young W. Kwon [3].

4 M. Sadiku. Elementos de Electromagnetismo $2^{\circ}$ Edición. Compañía Editorial Continental, S.A. México, 1998. p. 236.

5 Manuel Julio García. Lecture Notes on Numerical Analysis. Department of Mechanical Engineering, EAFIT University Medellin, Colombia. Enero de 2004. p. 64 . 
La solución analítica está fundamentada en el libro Elementos de Electromagnetismo de Sadiku [2].

\section{Desarrollo del tema}

\subsection{Cálculo del potencial electrostático en la región interior}

El potencial electrostático aparece en la literatura como una propiedad del campo eléctrico donde la derivada direccional del potencial electrostático indica la dirección de tendencia del campo eléctrico. La forma como se relacionan matemáticamente estas dos entidades puede expresarse así:

$$
\nabla V=-E
$$

Donde $\mathrm{V}$ es una función escalar que representa al potencial electroestático, E es el campo eléctrico y $\nabla$ es el operador gradiente.

La escogencia de esta representación se fundamenta en el hecho de que los campos eléctricos gozan de la propiedad de ser irrotacionales, y matemáticamente se conoce que el rotacional del gradiente de una función escalar es siempre cero ${ }^{6}$

$$
\nabla \times(\nabla V)=0
$$

Con estos antecedentes y considerando el hecho de que los campos eléctricos satisfacen la condición del flujo presentada por la ley de Gauss, de la forma:

$$
\nabla E=-\rho / \varepsilon_{r}
$$

Es directo el cálculo de (1) y (3) para obtener una relación entre el potencial electroestático y la densidad de carga, cuyo resultado corresponde a la ecuación de Poisson, como se puede apreciar:

\footnotetext{
6 M. Sadiku. Elementos de Electromagnetismo $2^{\circ}$ Edición. Compañía Editorial Continental, S.A. México, 1998. p. 157.
} 


$$
\nabla^{2} V=\rho / \varepsilon_{r}
$$

Donde $\rho$ es la densidad de carga y $\varepsilon_{\mathrm{r}}$ es la permitividad eléctrica del medio.

De acuerdo con la geometría del problema es adecuado hacer una representación del sistema en coordenadas cartesianas, correspondientes a la descripción de la ecuación de Poisson para este tipo de geometrías, que se expresa de la forma:

$$
\frac{d^{2} V(x, y)}{d x^{2}}+\frac{d^{2} V(x, y)}{d y^{2}}=\frac{\rho(x, y)}{\varepsilon_{0}}
$$

Las condiciones de frontera para el problema propuesto se expresan en la forma:

$$
\begin{aligned}
& V(x)=0, \forall x=0, \wedge, 0 \leq y \leq a \\
& V(x)=0, \forall x=b, \wedge, 0 \leq y \leq a \\
& V(y)=0, \forall 0 \leq x \leq b, \wedge, y=0 \\
& V(y)=V_{0}, \forall 0<x<b, \wedge, y=a
\end{aligned}
$$

Las cuales corresponden a las condiciones de frontera de Dirichlet.

El método del pesaje residual consiste en asumir una función de prueba que contiene coeficientes desconocidos por determinar. Para este fin, seleccionamos una función de peso $u(x, y)$ y se condiciona a que el promedio del pesaje residual sobre el dominio de la función sea cero.

$$
\int_{\Omega} u(x, y)\left(\frac{d^{2} V(x, y)}{d x^{2}}+\frac{d^{2} V(x, y)}{d y^{2}}-\frac{\rho(x, y)}{\varepsilon_{0}}\right) d \Omega=0
$$

Aplicando la extensión de Green al teorema de Gauss se obtiene una representación de la ecuación (7) que permite dividir el dominio en región interior y fronteras. Que se puede representar de la forma: 
$-\int_{\Omega}\left(\frac{d u(x, y)}{d x} \frac{d V(x, y)}{d x}+\frac{d u(x, y)}{d y} \frac{d V(x, y)}{d y}\right) d \Omega-\int_{\Omega} u(x, y) \rho(x, y) d \Omega+\int_{\Gamma_{n}} u\left(\frac{d V(x, y)}{d n}\right) d \Gamma=0$

La primera integral corresponde a la región interior del dominio, la segunda integral corresponde a la fuente de carga en el interior y la tercera integral corresponde al flujo de campo eléctrico en la frontera.

La solución al problema se simplifica para el propósito de este informe al considerar que la placa no presenta fuentes de carga. De esta forma la segunda integral en (8) se hace cero y la ecuación diferencial (4) se convierte entonces en la ecuación de Laplace, cuya representación en la forma débil es:

$-\int_{\Omega}\left(\frac{d u(x, y)}{d x} \frac{d V(x, y)}{d x}+\frac{d u(x, y)}{d y} \frac{d V(x, y)}{d y}\right) d \Omega+\int_{\Gamma_{n}} u\left(\frac{d V(x, y)}{d n}\right) d \Gamma=0$

La discretización del dominio en (9) se desarrolla usando elementos finitos bidimensionales triangulares lineales, para los cuales las variables de interpolación son lineales en $\boldsymbol{x}$ y $\boldsymbol{y}$ en la forma de:

$$
\omega=\omega(x, y)=a_{1}+a_{2} x+a_{3} y
$$

$\omega=\omega(x, y)=\left[\begin{array}{lll}1 & x & y\end{array}\right]\left\{\begin{array}{l}a_{1} \\ a_{2} \\ a_{3}\end{array}\right\}$

La aproximación de la solución depende de la selección de la función de prueba. En este proceso se deben determinar entonces los coeficientes desconocidos $a_{i}$ para lo cual la función de interpolación (10 - a) debe representar las variables nodales en los tres nodos del elemento triangular. Sustituyendo los valores de las coordenadas cartesianas de los nodos se obtiene:

$$
\omega_{e}=\left\{\begin{array}{l}
\omega_{1} \\
\omega_{2} \\
\omega_{3}
\end{array}\right\}=\left[\begin{array}{lll}
1 & x_{1} & y_{1} \\
1 & x_{2} & y_{2} \\
1 & x_{3} & y_{3}
\end{array}\right]\left\{\begin{array}{l}
a_{1} \\
a_{2} \\
a_{3}
\end{array}\right\}
$$


Desde donde es posible determinar los valores de las constantes $a_{i}$.

Para un elemento triangular la matriz del elemento es calculada de la forma:

$$
\left[K^{e}\right]=\int_{\Omega_{e}}\left(\frac{d u(x, y)}{d x} \frac{d V_{e}(x, y)}{d x}+\frac{d u(x, y)}{d y} \frac{d V_{e}(x, y)}{d y}\right) d \Omega
$$

a partir de funciones del tipo descritos en (11).

Donde $\mathrm{K}^{\mathrm{e}}$ es la matriz que contiene las pendientes de los planos evaluadas en cada nodo del elemento triangular ${ }^{7}$.

Cada elemento triangular posee nodos asociados con los otros elementos del dominio en cuya expansión del dominio se construye una matriz 'A' global del sistema que tiene dimensiones $n \times n$ siendo $n$ el número de nodos en el dominio.

El método de Galerkin selecciona las funciones $u$ y $V$ en el mismo espacio de funciones. La representación abstracta de la ecuación (12) es expresada como:

$$
a(V, u)=\ell(u)
$$

Cuya expansión a todo el conjunto de funciones en el dominio puede generalizarse de la manera siguiente:

$$
\langle A V, u\rangle=\langle\ell, u\rangle
$$

De acuerdo con las propiedades del producto interno (14) puede rescribirse en la forma:

$$
\langle A V-\ell, u\rangle=0
$$

Ya que $u$ es solución del sistema, en consecuencia la solución no trivial queda de la forma:

$$
A V-\ell=0
$$

7 Kwon, Young W; Bang, Hyocoong. The Finite Element Method Using Matlab. 1997.

Pag. 90. 
El segundo término de (16) corresponde al término de los flujos que entran a la región por la frontera de (9) y estarían descritos en las condiciones de frontera de Von Newman, no tratados en este documento debido a que no se consideran fuentes externas de campo eléctrico. Así al término restante de (16) se aplican las condiciones de frontera de Dirichlet y finalmente se resuelve el sistema de ecuaciones lineales para conseguir el valor del potencial en los nodos donde este valor es desconocido y ordenar el arreglo de valores de manera que el valor del potencial sea asignado al nodo correspondiente.

Los resultados obtenidos pueden apreciarse gráficamente en la figura (1). En ésta, el relieve de la región y el colorido representan el valor del potencial. En primera instancia no parece haber gran discrepancia entre las figuras izquierda y derecha, aparte de tres picos en la solución analítica.
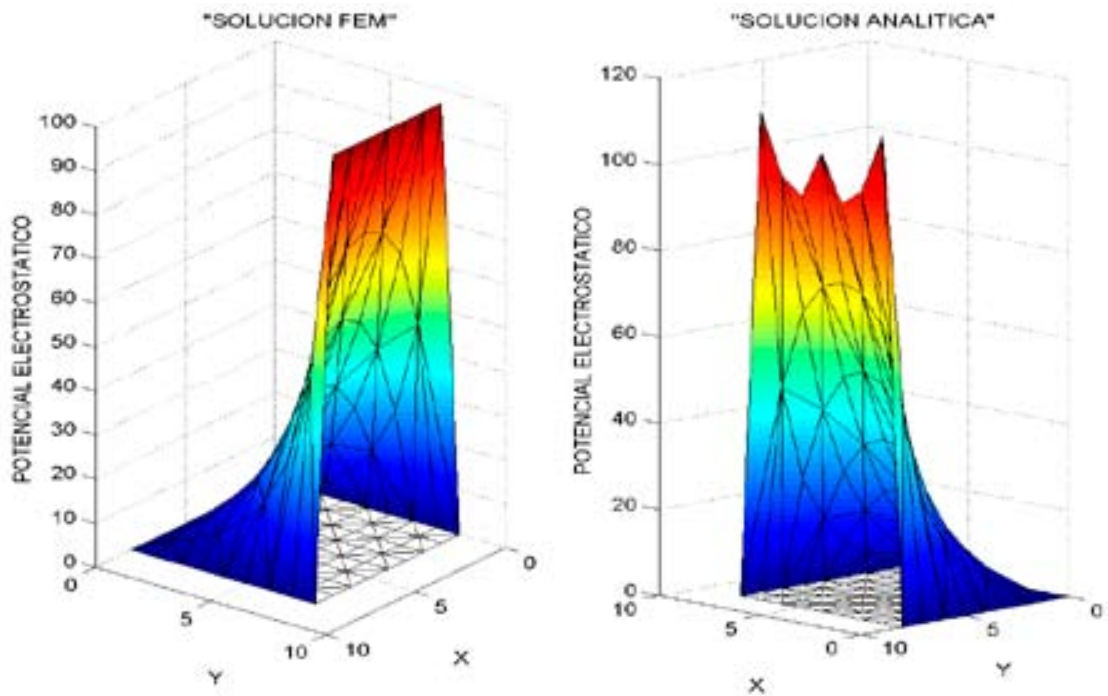

Figura 1. Distribución del POTENCIAL ELECTROStÁtico 
La solución analítica más usual es determinada mediante la solución de la ecuación diferencial (5). Al evaluar en ésta las condiciones de frontera de Dirichlet descritas en (6) se obtiene

$$
V(x, y)=\frac{4 V_{o}}{\pi} \sum_{n=1,3,5}^{\infty} \frac{\operatorname{sen}\left(\frac{n \pi x}{b}\right) \operatorname{senh}\left(\frac{n \pi y}{a}\right)}{n \cdot \operatorname{senh}\left(\frac{n \pi a}{b}\right)}
$$

y su representación grafica punto a punto en un dominio más puntual corresponde a la figura (2).

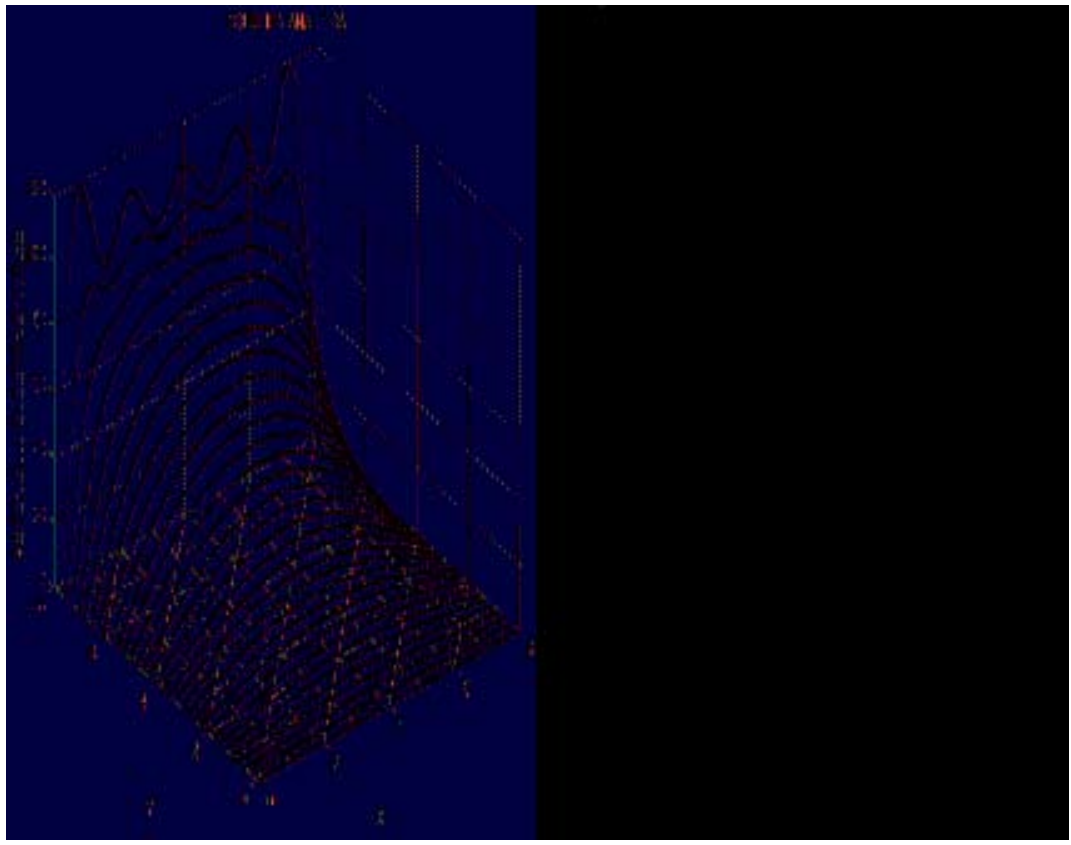

Figura 2. SoluCión AnALITICA

No está por demás resaltar el hecho de que ambas soluciones, la FEM y la analítica, son aproximaciones numéricas, sin embargo la solución FEM calculada en este ejercicio está más acorde con las 
condiciones iniciales del problema. Los picos que aparece en la parte derecha de la figura 1, corresponde a los valores calculados por la ecuación senosoidal 17 para las coordenadas correspondientes a cada nodo del dominio, y como puede apreciarse de la figura 2 , la solución analítica es más rizada en el extremo más alejado por efecto de la aproximación senosoidal para el valor del potencial en esa frontera.

Un indicativo numérico de las aproximaciones obtenidas por el método en cuestión es dado por el porcentaje de error calculado respecto a la norma. Éste se determina a partir de los valores del potencial electrostático calculado por los dos métodos de solución (FEM y analítico), calculando la norma de la diferencia entre los vectores en comparación dividida por la norma del vector de referencia, que en este caso corresponde a 'Va', la solución analítica ya que para efectos del problema se ha considerado la solución FEM como la función de prueba del sistema ' $\mathrm{V}$ ', aquí ‘ '*' indica la norma de referencia

$$
\% E\left(1_{*}\right)=\frac{\left\|V-V_{a}\right\|_{*}}{\left\|V_{a}\right\|_{*}}
$$

Tabla 1. Porcentajes de eRror entre la solución analítica y SOlución FEM

\begin{tabular}{|c|c|}
\hline$\%$ Error $(1 \infty)$ & 18.2759 \\
\hline \% Error $\left(l_{2)}\right)$ & 5.7674 \\
\hline
\end{tabular}

$\%$ Error l $\infty$ : indica el porcentaje de error con respecto a la norma infinita 8 .

$\%$ Error $\mathrm{l}_{2}$ : indica el porcentaje de error con respecto a la norma euclidiana.

\subsection{Cálculo del gradiente del potencial}

El gradiente representa la magnitud y la dirección de la máxima rapidez de incremento espacial del potencial escalar V. En la ecuación (1) se puede ver que el gradiente del potencial aumenta en la dirección opuesta a la dirección del campo eléctrico.

8 Manuel Julio García. Lecture Notes on Numerical Analysis. Department of Mechanical Engineering, EAFIT University Medellin, Colombia. Enero de 2004. p. 21. 
Para este cálculo se parte de que la función que describe el potencial escalar puede escribirse en cada región del dominio de la forma

$$
V(x, y)=\sum_{i} V_{i}(x, y) \omega(x, y)
$$

Y su gradiente en consecuencia

$$
\nabla V(x, y)=\frac{\partial V(x, y)}{\partial x} \widehat{i}+\frac{\partial V(x, y)}{\partial y} \hat{j}
$$

Reemplazando (17) en (18) se tiene

$$
\nabla V(x, y)=\sum_{i} V_{i}(x, y) \frac{\partial \omega(x, y)}{\partial x} \widehat{i}+\sum_{i} V_{i}(x, y) \frac{\partial \omega(x, y)}{\partial y} \widehat{j}
$$

El valor de $V_{i}(x, y)$ corresponde al valor del potencial calculado en la sección anterior para el nodo $i$ el cual es una constante calculada en la sección 2.1, de manera que en la ecuación (20) sólo queda calcular la derivada parcial de la función base descrita en la ecuación (10) para cada elemento. De allí puede verse que para un elemento lineal triangular

$$
\begin{aligned}
& \frac{\partial \omega_{i}(x, y)}{\partial x}=a_{2 i} \\
& \mathrm{y} \\
& \frac{\partial \omega_{i}(x, y)}{\partial y}=a_{3 i}
\end{aligned}
$$

Reemplazando las ecuaciones (21) en la (20) se obtiene:

$$
\begin{aligned}
& \sum_{i} V_{i}(x, y) \frac{\partial \omega(x, y)}{\partial x} \hat{i}=\sum_{i} V_{i}(x, y) a_{2 i} \hat{i} \\
& \sum_{i} V_{i}(x, y) \frac{\partial \omega(x, y)}{\partial y} \hat{i}=\sum_{i} V_{i}(x, y) a_{3 i} \hat{j}
\end{aligned}
$$


Ecuaciones que corresponden a la suma direccional de los gradientes de los nodos en cada elemento triangular en las respectivas coordenadas. A continuación se asigna el valor del promedio del gradiente del elemento triangular a cada nodo del mismo elemento. Al final cada coordenada nodal tendrá e número de gradientes asignados como el número de elementos triangulares que compartan dicho nodo. Para efectos de determinar el gradiente en cada nodo se calcula el promedio de los gradientes asignados a éste.

En la parte izquierda de la figura (3) es apreciable la distribución de los gradientes en el dominio y en la parte derecha se muestra la distribución del potencial ilustrado por el color asignado.
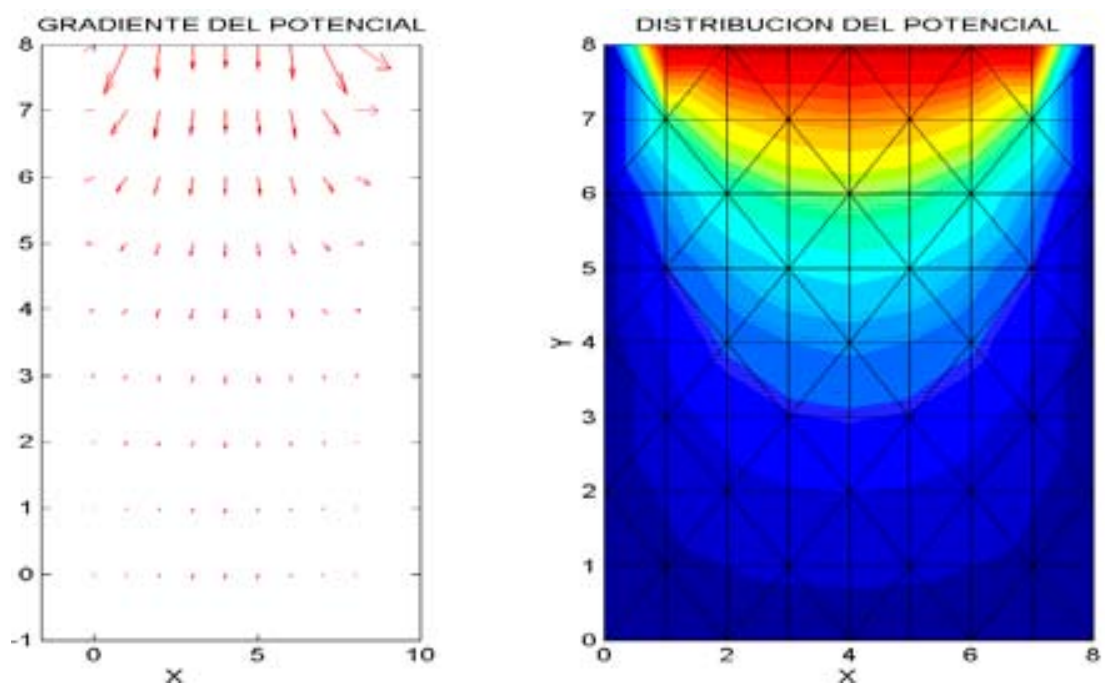

Figura 3. LÍNEAS DE CAMPO ELÉCTRICO Y DISTRIBUCIÓN DEL POTENCIAL ELECTROSTÁTICO 


\section{Conclusiones}

Las diferencias entre la solución analítica y la solución FEM observadas en la tabla (1) donde aparecen los porcentajes de error en los nodos cuyos resultados calculados por ambos métodos no son coincidentes, dan a entender una discrepancia debido a la aproximación senoidal de la función analítica.

El error calculado se ha realizado con base en dos soluciones que son aproximaciones y que, así la solución FEM esté más acorde con las condiciones iniciales del problema con respecto a la solución analítica que es una función armónica senosoidal, también demuestra que por efectos del pesaje residual o mínimo residuo esto afecta los resultados del sistema.

Lo que se puede extraer de este resultado es que el método de los elementos finitos para la solución al problema de la distribución del campo electrostático, arroja resultados consistentes con respecto a otros métodos ampliamente discutidos en la literatura técnica y científica.

\section{REFERENCIAS}

[1] Manuel Julio García Ruiz. Lecture Notes on Numerical Analysis, 2008. Fondo Editorial Universida Eafit. Department of Mechanical Engineering, EAFIT University, Medellin, Colombia.

[2] M. Sadiku (1998). Elementos de Electromagnetismo $2^{\circ}$ Edición. Compañía Editorial Continental, S.A. México.

[3] Kwon, Young W; Bang, Hyocoong. The Finite Element Method Using Matlab. Boca Raton: Crc Press, 1997. 
